\title{
Elegant and Practical Method of Fir Decimation Using Comb Filters in the Field of Digital Signal Processing
}

\author{
S. Arun Kumar ${ }^{1 *}$, P. Ganesh Kumar² \\ ${ }^{1}$ Department of Electronics and Communication Engineering, PSNA College of Engineering and Technology, \\ Dindigul, India \\ ${ }^{2}$ KLN College of Engineering, Pottapalayam, Tamil Nadu, India \\ Email: "murphy_arun@yahoo.co.in
}

Received 17 March 2016; accepted 10 April 2016; published 22 July 2016

Copyright $@ 2016$ by authors and Scientific Research Publishing Inc.

This work is licensed under the Creative Commons Attribution International License (CC BY).

http://creativecommons.org/licenses/by/4.0/

cc) (i) Open Access

\section{Abstract}

This paper deals with the technology of using comb filters for FIR Decimation in Digital Signal Processing. The process of decreasing the sampling frequency of a sampled signal is called decimation. In the usage of decimating filters, only a portion of the out-of-pass band frequencies turns into the pass band, in systems wherein different parts operate at different sample rates. A filter design, tuned to the aliasing frequencies all of which can otherwise steal into the pass band, not only provides multiple stop bands but also exhibits computational efficiency and performance superiority over the single stop band design. These filters are referred to as multiband designs in the family of FIR filters. The other two special versions of FIR filter designs are Halfband and Comb filter designs, both of which are particularly useful for reducing the computational requirements in multirate designs. The proposed method of using Comb FIR decimation procedure is not only efficient but also opens up a new vista of simplicity and elegancy to compute Multiplications per Second (MPS) and Additions per Second (APS) for the desired filter over and above the half band designs.

\section{Keywords}

Decimation, Multiband, Halfband, Comb Filter, MPS, APS, Multirate Signal Processing

\section{Introduction}

In many practical applications of digital signal processing, quite often we come across the problem of changing

\footnotetext{
${ }^{*}$ Corresponding author.
}

How to cite this paper: Arun Kumar, S. and Ganesh Kumar, P. (2016) Elegant and Practical Method of Fir Decimation Using Comb Filters in the Field of Digital Signal Processing. Circuits and Systems, 7, 2476-2488. 
the sampling rate of a signal by either increasing it or decreasing it to some extent. For example, in telecommunication practices that transmit and receive different types of signals, namely, teletype, facsimile, speech, video, etc., there is a requirement to process the various signals at different sampling rates commensurate with the corresponding bandwidths of the signal types.

The process of sampling a signal from a given rate to a different rate is called sampling rate conversion. Such sampling rate conversions are needed when systems with different signal frequencies and consequent different sampling rates are to be interconnected. Systems that employ multiple sampling rates in the processing of digital signals are called Multirate Digital Signal Processing Systems. In multirate systems the sampling frequency is changed during the signal processing. In most cases the sampling rates at the input and output ends differ rather markedly. In other cases the sampling rate is changed only internally while the input and output rates keep one and the same. This is done in order to improve the efficiency of the processing.

These techniques are commonly used in narrow band lowpass, highpass, bandpass filters, and filter banks as well as the so-called transmultiplexers such as the converters between FDM and TDM terminals [1]-[10]. Multirate processing provides the method for changing the sampling rate, and forms the basis for implementing complex signal processing applications with reduced computational requirements. Multirate processing is defined as a signal processing implementation that uses more than one sampling rate to perform the desired digital system operations. The two basic operations performed are decimation and interpolation. Decimation results in sample rate decrease while interpolation provides a sample rate increase.

$$
\text { Decimation }=\text { Filtering }(\text { anti-aliasing })+\text { Downsampling }
$$

\section{Decimation with a Factor $M$}

The process of reducing the sampling frequency is called decimation-even if the reduction is not by a factor of ten. Decimation is often used in A/D converters. The analog signal is sampled with a very high sampling frequency in order to reduce the requirement of the anti-aliasing filter. Usually, the signal frequency is oversampled by a factor that is larger power of two. The digital signal is then bandlimited by a digital filter with a stopband angle slightly less than $\pi / 2$. The sampling frequency can therefore be reduced by a factor of two by simply dropping every other sample and is repeated until the desired sample rate is obtained. The relation between the Fourier transforms of the decimated signal and the original signal is

$$
X_{d}\left(\mathrm{e}^{j \omega T}\right)=\frac{1}{M} \sum_{k=0}^{M-1} X\left(\mathrm{e}^{j(\omega T-2 \pi k) / M}\right)
$$

where $M$ is the decimation factor.

Downsampling (decimation) by an integer factor of $M$ means taking one sample from the data sequence $x(n)$ for every $M$ samples and discarding the last $M-1$ sample. It requires a decimation (anti-aliasing) filter to avoid aliasing before downsampling. Two-stage decimation can dramatically reduce the anti-aliasing filter length [11] [12].

The rest of this paper is organized as follows. Section 2 deals with the multiband FIR design technique and provides the attitude of passband and stopband. Section 3 deals with the half band FIR design technique which describes the MPS, APS and storage requirements. Section 4 describes the Comb FIR filter. Section 5 is an example design for Comb FIR decimation filter. Section 6 discusses the results and derives an inference. Finally we have summarized the conclusion in Section 7.

\section{Multiband FIR Design}

Multiband designs take advantage of regions that will not alias into the passband. These regions are called "don't care regions". The multiband design is specified as one passband with multiple stopbands and "don't care regions”. The multiple stopbands are chosen to attenuate all frequencies that would fold into the passband. The multiband design requires a filter with passband $f_{p}$ and data decimation factor $D=10$. These constraints of multiband frequency response require a design optimization technique that allows the multiple band constraints to be specified [13] [14]. The Parks-McClellan designs can result in unacceptable performance in the transition regions; therefore the frequency response of the filter should always be analyzed to ensure the design solution which satisfies the requirements. The multiple stopband regions for the $\mathrm{k}^{\text {th }}$ multirate stage are defined by the equation, 


$$
S B_{n}(k)=\frac{n F}{\prod_{i=1}^{k} D(i)} \pm f_{p} \quad \text { where } n=1,2,3, \cdots, \text { Int }(D(k) / 2)
$$

Accordingly the regions outside the passband and not defined by Equation (2), represent the "don't care bands" (i.e., $\phi$-bands). Note that the number of $\phi$-bands is equal to the integer portion of $(D(k)+1) \div 2$. For $D$ an even integer, the upper side band region extends to one half the input sampling rate including only the negative portion of $f_{p}$ in Equation (2).

For the two-stage multirate filter to determine the multi stop band filter stages with the given specifications.

The passband frequency $f_{p}=100$

Passband ripple $\delta_{p}=0.1$

Stopband ripple $\delta_{s}=0.001$

$$
\begin{aligned}
& S B_{n}(1)=\frac{n F}{\pi D_{i=1}^{k}(i)} \pm f_{p}=\frac{25000}{200} \pm 100 \\
& S B_{n}(1)=1250 n \pm 100
\end{aligned}
$$

For the stage 1 filter, which precedes a decimation of 20, the ten stopbands are defined as

$$
S B_{n}(1)=1250 n \pm 100 \quad \text { where } n=1,2,3, \cdots, 10
$$

where the tenth stop band is stopped at one half the sampling rate. For the stage 2 filter, which precede a decimation of five, two stop bands are given by

$$
S B_{n}(2)=250 n \pm 100 \text { where } n=1,2 .
$$

There are no simple formulae for determining the order required to achieve the multiband filter requirements. The researchers can turn to a trial-and-error process using the conventional PM filter order as a starting point and trying an arbitrary 25\% reduction. If a satisfactory design is achieved, then proceed with $35 \%$. If the $25 \%$ does not work then step back and proceed with $15 \%$ and so on and so forth. By performing many multiband design trends in the modified order as a function of the passband, the decimation factor can be finally established. The savings can be significant for cases where they don't care regions represent a high percentage of the out-of-passband frequency range. This occurs for cases when the passband is small compared to the sampling rate and also when moderate decimation factors are desired. Negative results sometimes occur for lower $\phi$-band percentage as much as for high decimation factors. Apparently, more zeros are required to adapt to several stopbands than the conventional design in the negative cases.

The percentage portion of the $\phi$-bands in the transition width and stopband for multiband stage $k$ is given by the equation,

$$
\phi \text {-band }(k)=\left[1-\frac{(D(k)-1) * f_{p}}{\frac{F}{2 \prod_{i=1}^{k} D(i-1)}-f_{p}}\right] \times 100 \quad D(0)=1
$$

We designed the filters from illustration and found that they did not result in a better design. The stage 1 filter design required a 59 order filter versus a 55 order filter for conventional PM low pass design.

This demonstrates the need to design the filters in order to obtain the most accurate estimate possible. Likewise for the stage 2 filter, a 55 order multiband design was required versus a 55 order conventional design; this resulted in a stopband attenuation level of $59.87 \mathrm{~dB}$ versus $60 \mathrm{~dB}$ specified. The designer would have to assess the impact of this on the system to determine if a higher order design was required.

The use of trend and/or design curves has been popular in DSP design and analysis. For our multiband design problems, many designs can be tried and evaluated interactively until an acceptable design is achieved. The trend can be embedded into the program and used to select the initial filter order based on the $\phi$-band percentage. 
Design Example for Multiband Filter:

To demonstrate the effectiveness of using a multiband filter to decimate a speech signal, the following design requirements and solutions were given

$$
\begin{aligned}
& f_{p}=3000 \mathrm{~Hz} \text { (Pass Band Frequency) } f_{s}=5000 \mathrm{~Hz} \text { (Stop Band Frequency) } \\
& \delta p=(0.1 \mathrm{~dB}) \quad \text { (Pass Band Ripple) } \quad \delta s=(-50 \mathrm{~dB}) \quad \text { (Stop Band Ripple) } \\
& F=64000 \mathrm{~Hz} \text { (Sampling frequency) } \quad D=8\left(\text { Decimation factor }=\frac{\text { Input Sampling rate }}{\text { Output Sampling rate }}\right)
\end{aligned}
$$

$N=$ Order of the filter

Solution:

Peled and Liu found that the conventional lowpass design required a 160 order filter to meet the above specifications. This results in the following computational and storage requirements.

After using the conventional 160 order filter, the results are as follows.

$$
\begin{aligned}
& \text { MPS }=F(N+1) / 2 D=64000(160+1) / 2 \times 8 \\
& =644000 \text { (Multiplications per second) } \\
& \text { APS }=F(N-1) / D=64000(160-1) / 8 \\
& =1272000 \text { (Additions per second) } \\
& \text { Storage }=2(160)+1=321
\end{aligned}
$$

They found that a multiband filter of order 80 met the same requirements. The computational and storage requirements are reduced by a factor of two.

$$
\begin{aligned}
& \text { MPS }=324,000 \rightarrow 49.7 \% \text { savings } \\
& \text { APS }=632,000 \rightarrow 50.3 \% \text { savings } \\
& \text { Storage }=161 \rightarrow 49.8 \% \text { savings }
\end{aligned}
$$

We computed the percentage portion of the $\phi$-bands in the "don't care regions" relative to the stop band for the filter design.

$$
\text { Percentage of } \phi \text {-band }=\left(1-\frac{7 \times 3000}{32000-3000}\right) \times 100=27.6 \%
$$

Consequently, a significant percentage gain was realized with a relatively large pass-band. The designer must assess the significance of the savings for the application and decide whether a multiband design is necessary.

The foregoing results indicate that the conclusions reached in the previous section on the best multirate implementation may be altered if the multiband designs are taken into account. Therefore it is cautioned that the approaches developed herein should be used as a guide only and that to reach a final design, several factors must be considered.

The "don't care regions" can exhibit rather large amplitude levels compared to the passband response; therefore the overall output noise could be significantly effected. This aspect must be analyzed to ensure that the system performance is not degraded.

\section{Halfband FIR Design}

Halfband FIR filters have the unique property that all the even coefficients of the filter are zero. Therefore, the number of computations and storage for a $k$ stage filters are

$$
\begin{gathered}
\text { Multiplications }=[N(k)+5] / 4 \\
\text { Additions }=[N(k)+5] / 2 \\
\text { Storage }=[3 N(k)+5] / 2
\end{gathered}
$$

which represents a reduction by approximately a factor of two. The storage required for coefficients is given by Equation (11). The computation rates are given by 


$$
\begin{aligned}
& \operatorname{MPS}(k)=F(k) *[N(k)+5] / 4 \\
& \operatorname{MPS}(k)=F(k) *[N(k)+1] / 2
\end{aligned}
$$

The passband and stopband have equal ripple factors. The stop band width is equal to the passband width, and that the symmetry point is at $0.25 \mathrm{~F}$, where the power is at 0.5 . Therefore the halfband constraints for stage $k$ of a $K$-stage multiband filter are defined by

$$
\begin{gathered}
\delta_{h b}(k)=\delta_{p}(k)=\delta_{s}(k)=\operatorname{MIN}\left(\frac{\delta_{P}}{K}, \delta_{s}\right) \quad k=1,2, \cdots, K \\
f_{p}(k)=f_{p} \\
{\left[\frac{F}{\prod_{i=1}^{k} D(i)}\right]-f_{p}(k)=F(k)-f_{p}}
\end{gathered}
$$

These criteria allow aliasing to occur in the final transition band. For the case where no aliasing is allowed in the final transition band, the passband is extended to meet the requirement of no aliasing.

$$
f_{p}(k)= \begin{cases}\frac{F(K)}{2} & k=1,2, \cdots, K-1 \\ f_{p} & k=K\end{cases}
$$

The stopband critical frequency is expressed by

$$
f_{s}(k)=\frac{F}{2^{k}}-f_{p}(k) \quad k=1,2, \cdots, K
$$

Note that for the case where aliasing is not permitted in the final transition band $0.5 F(k)$, the last stage does not meet the halfband constraints (i.e., the passband length is not equal to the stopband length). Any power of two can be obtained by cascading halfband filters [15]. For cases that are not a power of two, only a portion of the multirate filter can be implemented using halfband filters. The number of stages, $K$, for a decimation $D$ is given by the equation,

$$
K=\log _{2}(D)
$$

Halfband filters are useful for decimating the data by a factor of 2 . This is a result of the sampling frequency being constrained to less than $0.5 \mathrm{~F}$ and the stopband limiting the aliasing level to $\delta_{s}$ in the passband. It can be shown that halfband filters are always odd-order filters. This is left as a problem. Also, since the even coefficients are zero, the only orders that need to be implemented are expressed by

$$
N_{h b}=3,7,11,15,19,23, \cdots
$$

Since the alternating odd values result in zero filter coefficients (i.e., 5, 9, 13, $\cdots)$, the filter design can specify these values, resulting in a better frequency response that can be implemented with the next lower odd-order halfband. Since the reduction in computations and storage implies that only a portion of the data can be processed, the hardware and software must have the intelligence to perform the operation. This will require special software code and/or hardware complexity to address the proper data and perform the halfband algorithm. This could offset the advantages gained by the reduced computations required and must be included in the overall design process.

\begin{tabular}{lllllllllllllllllllllllllll}
\hline$n$ & -10 & -9 & -8 & -7 & -6 & -5 & -4 & -3 & -2 & -1 & 0 & 1 & 2 & 3 & 4 & 5 & 6 & 7 & 8 & 9 & 10 \\
$h_{o}$ & 0 & $\mathrm{~h}_{9}$ & 0 & $\mathrm{~h}_{7}$ & 0 & $\mathrm{~h}_{5}$ & 0 & $\mathrm{~h}_{3}$ & 0 & $\mathrm{~h}_{1}$ & $\mathrm{~h}_{0}$ & $\mathrm{~h}_{1}$ & 0 & $\mathrm{~h}_{3}$ & 0 & $\mathrm{~h}_{5}$ & 0 & $\mathrm{~h}_{7}$ & 0 & $\mathrm{~h}_{9}$ & 0 & 0 \\
\hline
\end{tabular}

Design Example for Halfband Filter:

To design a multirate filter system using half band filter designs to effect a decimation of 32 with the follow- 
ing specifications.

$$
f_{p}=50 \mathrm{~Hz}, \quad \delta_{p}=0.01, \quad \delta_{s}=0.001, \quad F=4800 \mathrm{~Hz}, \quad D=32
$$

Allow aliasing in the final transition band.

Design method: Parks-McClellan (PM) filter approximation.

Design procedure:

Step 1: Determine the number of stages.

Step 2: Filter specifications.

Step 3: Computation of filter orders required for each stage.

Step 4: Computational and storage requirements for the halfband design.

$$
\begin{gathered}
K=\log _{2}(32)=5 \\
\delta_{h b}(k)=\delta_{p}(k)=\delta_{s}(k)=\operatorname{MIN}\left(\frac{0.01}{5}, 0.001\right)=0.001 \\
f_{p}(k)=50 \\
f_{s}(k)=\left[\begin{array}{c}
\left.\frac{4800}{\prod_{i=1}^{k} D(i)}\right]-50=F(k)-50
\end{array}\right.
\end{gathered}
$$

\begin{tabular}{|c|c|c|c|c|c|c|}
\hline \multirow{2}{*}{ Stage $k$} & \multicolumn{2}{|c|}{ Stage Sampling Rate } & \multirow{2}{*}{ Stopband Frequency $f_{s}(k)$} & \multirow{2}{*}{ Transition Width } & \multirow{2}{*}{ Filter Order $N(k)$} & \multirow{2}{*}{$\begin{array}{l}\text { Halfband } \\
\text { Filter Order }\end{array}$} \\
\hline & Input $F(k-1)$ & Output $F(k)$ & & & & \\
\hline 1 & 4800 & 2400 & 2350 & 0.479 & 7.7 & 9 \\
\hline 2 & 2400 & 1200 & 1150 & 0.458 & 8.0 & 9 \\
\hline 3 & 1200 & 600 & 550 & 0.417 & 8.7 & 9 \\
\hline 4 & 600 & 300 & 250 & 0.333 & 10.6 & 11 \\
\hline 5 & 300 & 150 & 100 & 0.167 & 20.3 & 21 \\
\hline
\end{tabular}

The Table 1 depicts that the output sampling rate is half of the input sampling rate. In the proposed method, at stage 5, stop band frequency and transition width decreased rather than the existing method. This is plotted in Figure 1.

Table 1. Halfband filter requirements (vide Figure 1).

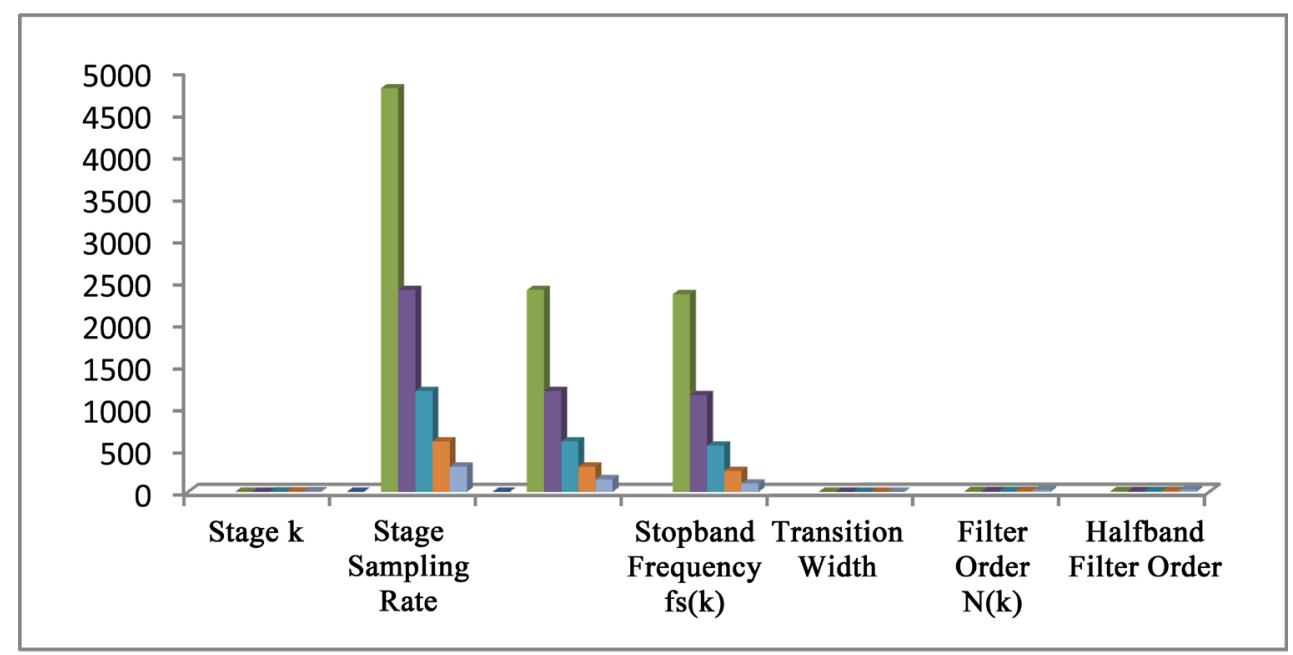

Figure 1. Halfband filter requirements as in Table 1. 
Simultaneously conventional filter order and half band order were also increased in the proposed method. The conventional filter orders were calculated using the simple form of the PM filter approximation. Next we computed the computational and storage requirements for the half band design and compared it to the single stage implementation. These results are presented in Table 2.

In the proposed method at the stage 5, when the half band filter order is increased, automatically the MPS and APS have decreased but the storage has increased. These are all plotted in Figure 2.

The single stage design results are, as follows:

$$
N=243, \quad \text { MPS }=18300, \quad \text { APS }=36300, \quad \text { Storage }=487
$$

This requires higher computations and significantly larger storage. Several other options are available for implementing this multirate filter, including two-stage and three-stage designs. Some of these options are summarized in Table 3.

In the proposed method at stage 3, D (1) $=4, \mathrm{D}(2)=4$, and $\mathrm{D}(3)=2 \mathrm{H}(\mathrm{B})$, while the MPS and APS have increased, the storage has decreased. This is plotted in Figure 3.

And compared to that of existing method [16] [17]. These designs in general result in less computational requirements than the total half band implementation. Note that the lowest computational requirements result for the three-stage design, with the last two stages designed as half band stages. Again, this example is provided to illustrate the importance of trading off potential implementations. We did not design the filters, and therefore the results could change slightly owing to inaccuracies in the filter order estimates. Also, the complexity in hardware and software must ultimately be assessed, including finite arithmetic effects, before a final selection is made.

\section{Comb FIR Design}

A simple FIR filter is constructed by averaging $N$ samples (i.e., $h(n)=1 / N$ for $n=1,2, \cdots, N$ ). This class of FIR

Table 2. Half band computation and storage requirements (vide Figure 2).

\begin{tabular}{ccccc}
\hline & Halfband Filter Order & \multicolumn{3}{c}{ Computations } \\
\cline { 3 - 5 } Stage $\boldsymbol{k}$ & MPS & APS & Storage \\
\hline 1 & 9 & 8400 & 12,000 & 16 \\
2 & 9 & 4200 & 6000 & 16 \\
3 & 9 & 2100 & 3000 & 16 \\
4 & 11 & 1200 & 1800 & 19 \\
5 & 21 & 975 & 1650 & 34 \\
Total & & 16,875 & 24,450 & 101 \\
\hline
\end{tabular}

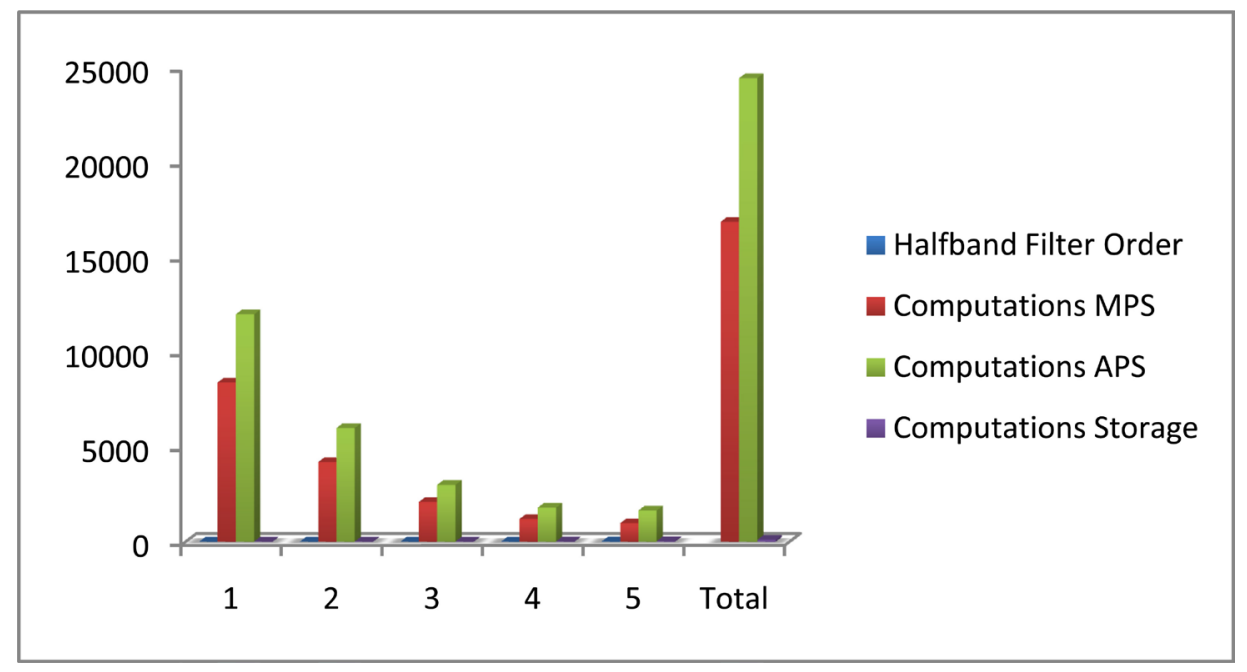

Figure 2. Half band computation and storage requirements as in Table 2. 
Table 3. Alternative design implementations (vide Figure 3).

\begin{tabular}{ccccccc}
\hline \multirow{2}{*}{ Number of Stages } & \multicolumn{3}{c}{ Decimation } & \multicolumn{3}{c}{ Computational Requirements } \\
\cline { 2 - 7 } & D (1) & D (2) & D (3) & MPS & APS & Storage \\
\hline 2 & 16 & 2 & --- & 11,700 & 22,500 & 174 \\
2 & 8 & 4 & --- & 11,700 & 21,900 & 130 \\
3 & 16 & $2 \mathrm{HB}$ & --- & 11,175 & 21,450 & 169 \\
3 & 8 & 2 & 2 & 12,300 & 22,500 & 121 \\
3 & 4 & 4 & 2 & 14,100 & 24,900 & 109 \\
3 & 8 & $2 \mathrm{HB}$ & $2 \mathrm{HB}$ & 11,175 & 20,250 & 121 \\
\end{tabular}

Legend: HB = Halfband filter.

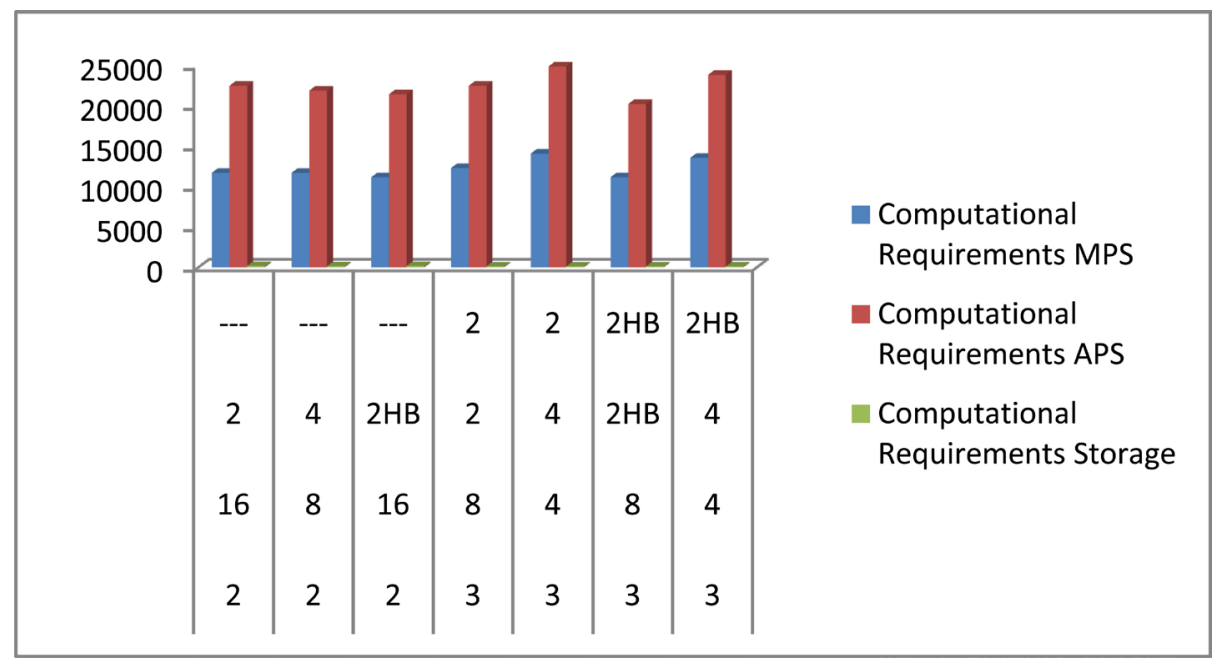

Figure 3. Alternative design implementations as in Table 3.

filter is called a Comb filter. The transfer function is given by

$$
H_{N}(z)=\frac{1}{N} \sum_{i=0}^{N-1} z^{-i}
$$

and the frequency response is given by

$$
H_{N}(f)=\mathrm{e}^{-J 2 \pi f(N-1) / F} \frac{\sin (\pi f N / F)}{N \sin (\pi f / F)}
$$

Figure 4 shows Comb filter frequency response $(N=10)$ which is obtained by using MATLB programming.

The Figure 4 shows that the response has one major lobe and many minor sidelobes, but if the desired passband is small compared to the input sampling frequency, the nulls in the response will reject the bands, which will fold into the passband for a decimator with $D=N$. There are

$N-1$ nulls in the response that occur at frequencies

$$
f_{n}=n F / N, \quad n=1,2, \cdots, N-1
$$

The allowance for the passband width is based on the required passband and stopband deviations. The response is evaluated at each stopband edge frequency and at the passband edge to assure that the requirements are fulfilled. The response constraints are expressed by

$$
S B_{n}(k)=\frac{n F(k-1)}{N(k)} \pm f_{p}, \quad n=1,2, \cdots, \operatorname{Int}(N(k) / 2)
$$




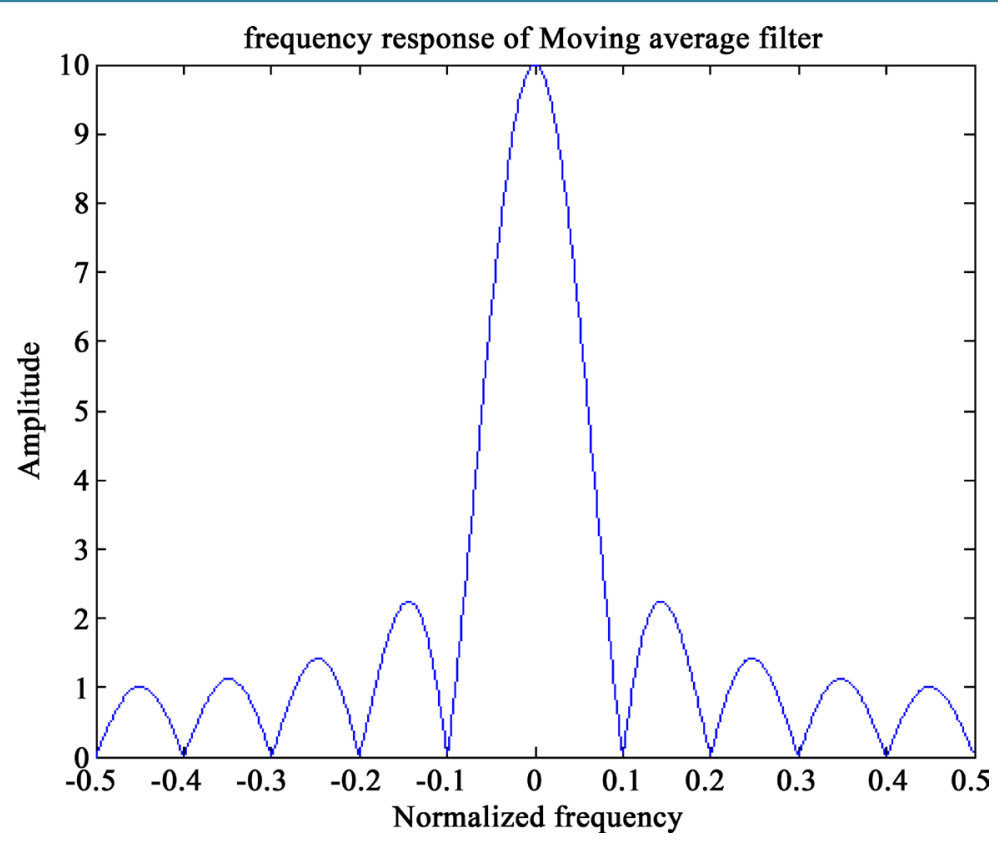

Figure 4. Comb filter frequency response $(N=10)$.

$$
\begin{gathered}
\left|H_{N}\left(S B_{n}(k)\right)\right| \leq \delta_{s} \\
1-\left(\delta_{p} / K\right) \leq\left|H_{N}\left(0 \rightarrow f_{P}\right)\right| \leq 1+\left(\delta_{P} / K\right)
\end{gathered}
$$

where $K$ is the number of stages in the multistage design. If the response does not meet the specifications, then either the filter requirements have to be relaxed or the decimation factor should be lowered until the criteria defined by Equation (26) is satisfied. These expressions are easily programmed, which provide an automated approach to evaluate the application of the Comb filter to multirate systems [18]-[21].

The Comb filters are most often used either as the first stage of a multistage decimator or the last of the multistage interpolator. Again we stress the need to evaluate the benefits of using the Comb filter versus the hardware and software requirements. The Comb filter requires the accumulation of $N$ inputs normalized by $N$. If $N$ is a power of two, the normalization can be implemented simply via shifts, and no multiplications are required. The worst-case implementation would require $N$ multiplies by the impulse response coefficients $1 / N$. Generally, the filter is implemented without multiplies and the normalization is accounted for via scaling the input to the nearest power of two that avoids overflow expressed by,

$$
r=\left\langle\log _{2}(N)\right\rangle
$$

The resultant value can then be multiplied by the factor $2^{r} / N$ to provide the exact normalization required. In many cases, this multiplication can be neglected or compensated for in the subsequent operations. The computation and storage requirements, assuming an implementation requiring one multiple, are expressed by

$$
\begin{gathered}
\operatorname{MPS}(k)=F(k-1) / N(k)=F(k) \\
\operatorname{APS}(k)=F(k-1) *(N(k)-1 / N(k)) \\
\text { Storage }(k)=N(k)+1
\end{gathered}
$$

We assumed that one register was required to hold the partial sum as the operation is performed.

\section{Design Example for Comb Filter}

Design the anti-aliasing FIR filter or decimation filter [22].

Given a DSP downsampling system with the following specifications: 
Sampling rate $=6000 \mathrm{~Hz}$

Input audio frequency range $=0-800 \mathrm{~Hz}$

Passband ripple $=0.02 \mathrm{~dB}$

Stopband attenuation $=50 \mathrm{~dB}$

If the window method is used the parameters required are FIR filter length and cut-off frequency.

Solution:

Specifications are recognized as:

Anti-aliasing filter operating at the sampling rate $=6000 \mathrm{~Hz}=F$

Passband frequency range $=0-800 \mathrm{~Hz}\left(f_{p}\right)$

Stopband frequency range $=1-3 \mathrm{kHz}\left(f_{s}\right)$

Pass band ripple $=0.02 \mathrm{~dB}\left(\delta_{p}\right)$

Stop band attenuation $=50 \mathrm{~dB}\left(\delta_{s}\right)$

Filter type = FIR. In Figure 5 and Figure 6 .

The Hamming window is selected, since it provides $0.019 \mathrm{~dB}$ ripple in the pass band and $53 \mathrm{~dB}$ attenuation in the stop band. The normalized transition band is given by

$$
\Delta f=\frac{f_{\text {stop }}-f_{\text {pass }}}{F}=\frac{1000-800}{6000}=0.033
$$

The length of the filter and the cutoff frequency can be determined by

$$
N=\frac{3.3}{\Delta f}=\frac{3.3}{0.033}=100 .
$$

We choose the odd number, that is, $N=101$, and

$$
f_{c}=\frac{f_{\text {pass }}+f_{\text {stop }}}{2}=\frac{800+1000}{2}=900 \mathrm{~Hz} .
$$

\section{Results and Discussion}

\section{MATLAB and Gold Wave Support Tools}

Using Filter Design and Analysis (FDA) tool, the following characteristics were observed. The magnitude and phase response of the decimation filter sampled at the frequency of $6 \mathrm{kHz}$, are shown in Figure 7; its information signal is displayed in Figure 8 and Figure 9.

An ideal filter is often called a brick wall filter. The passband includes all those frequencies that go through

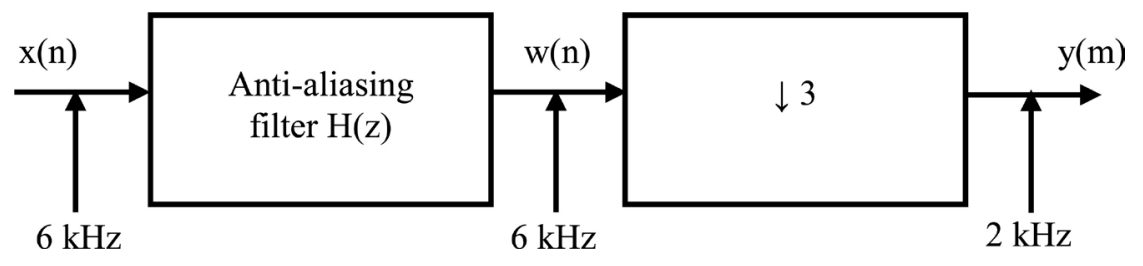

Figure 5. Design of the anti-aliasing FIR filer or decimation filter.

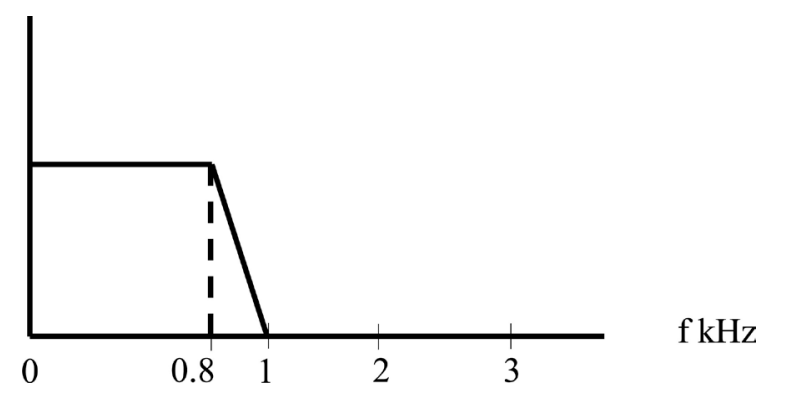

Figure 6. Ideal frequency response of an anti-aliasing FIR filer or decimation filter. 


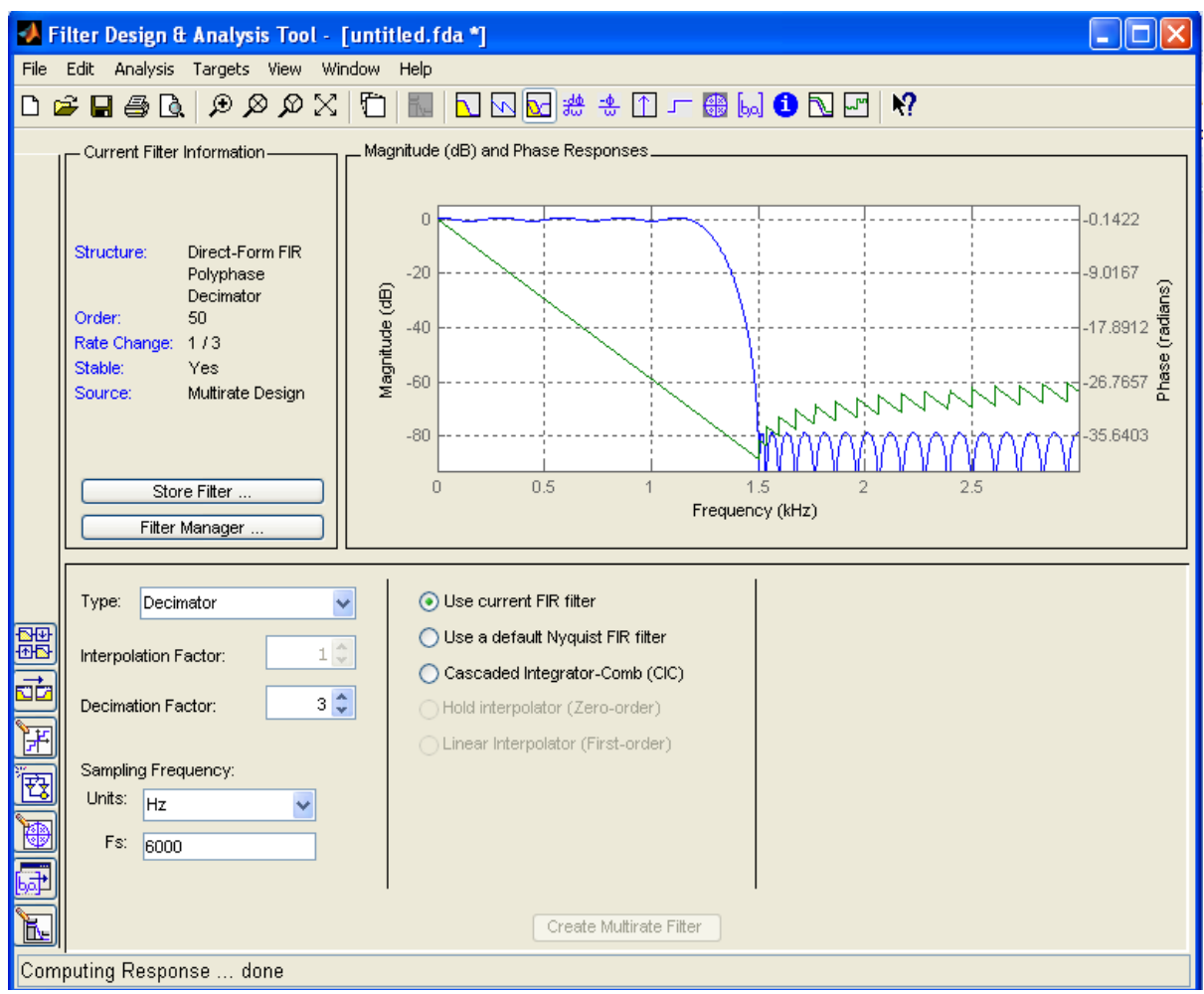

Figure 7. Magnitude and phase response of decimation filter sampled at the frequency of $6 \mathrm{kHz}$.

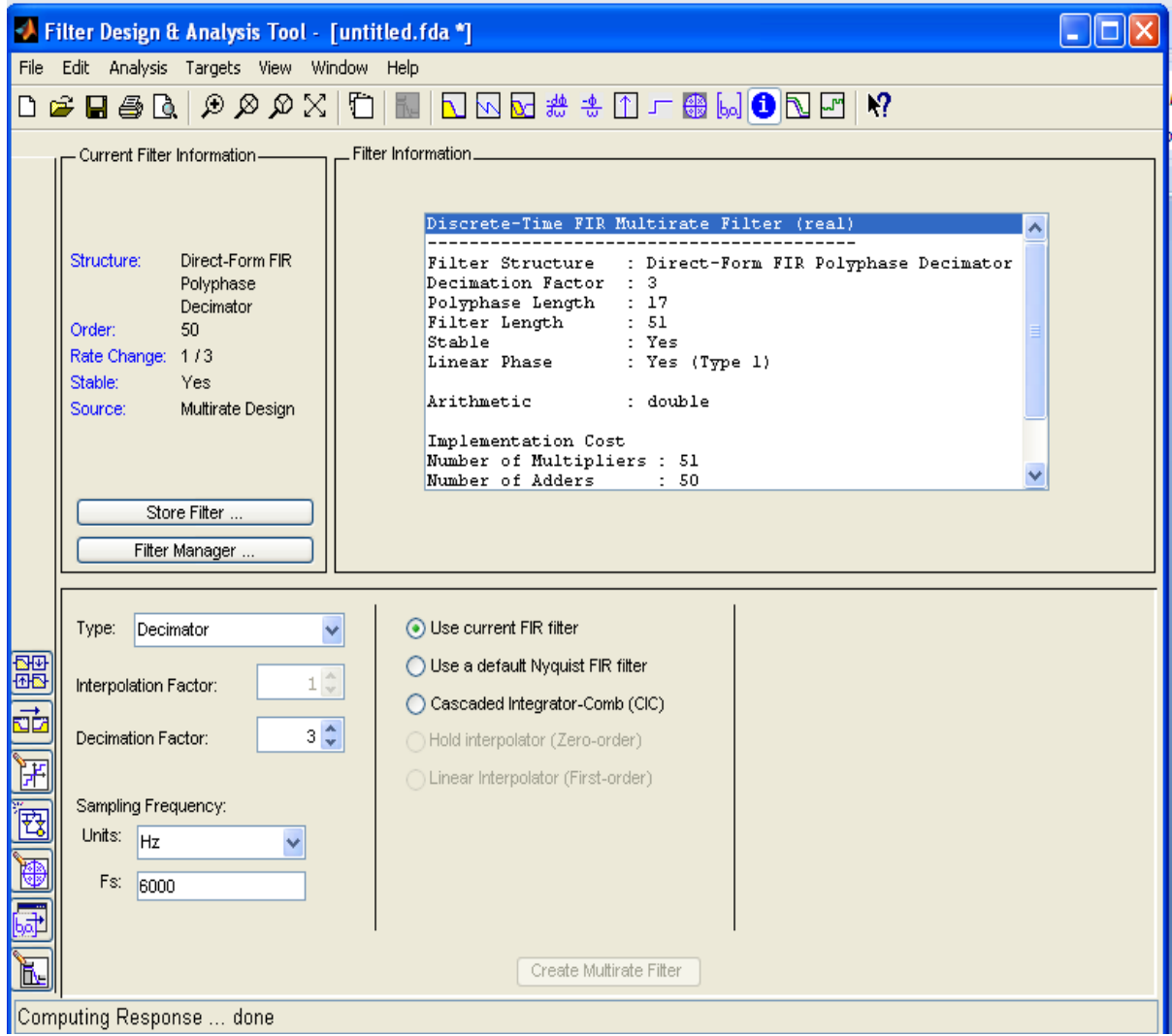

Figure 8. Information signal of decimation filter sampled at the frequency of $6 \mathrm{kHz}$. 


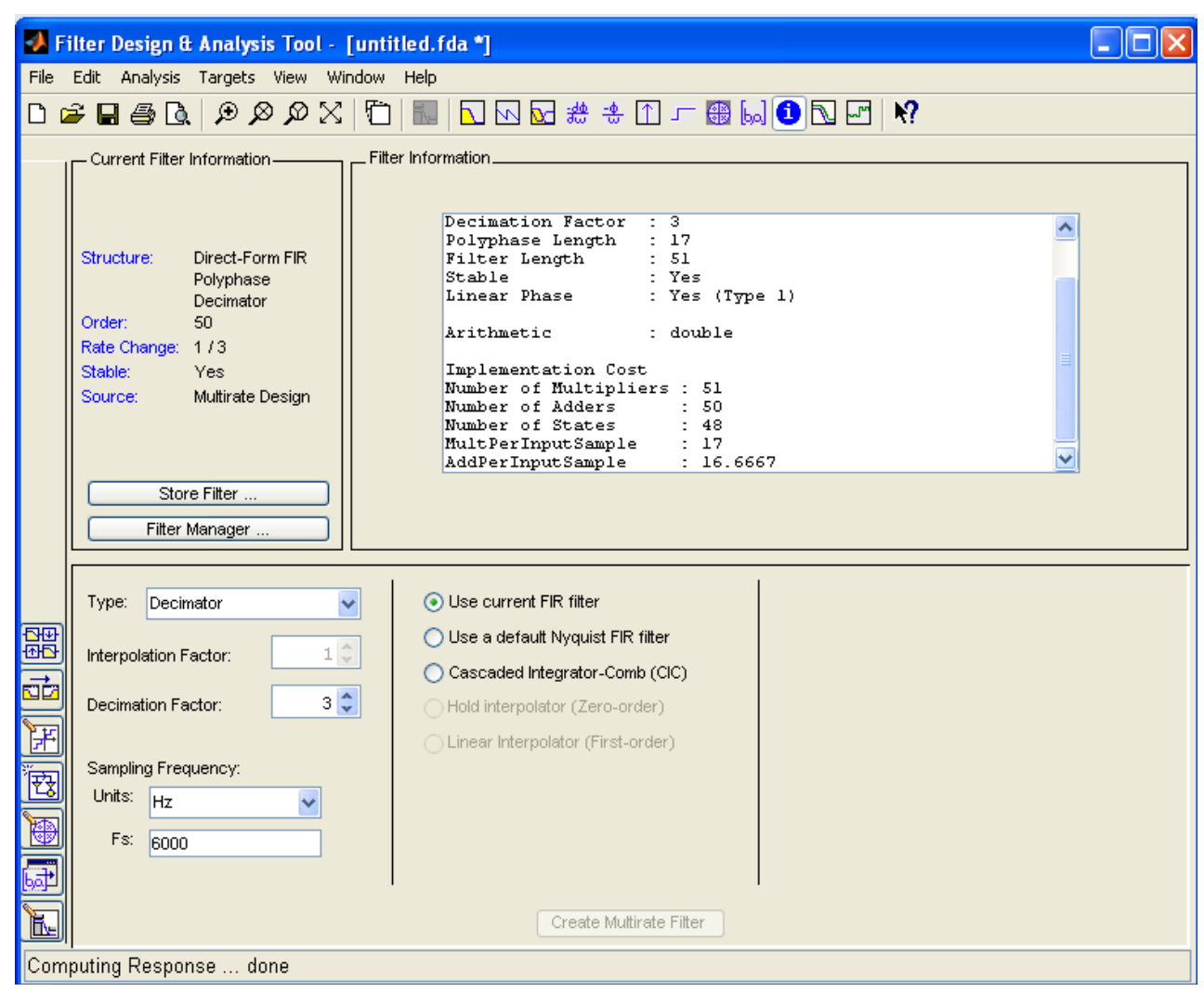

Figure 9. Information signal of decimation filter sampled at the frequency of $6 \mathrm{kHz}$.

the filter with no attenuation (the amplitude is maximum). The stopband includes all those frequencies that don't get through the filter (the amplitude is zero and the attenuation is infinite). Real filters differ from the ideal filter in some way or other as follows.

There could be ripple in the pass band as well as in the stop band.

There could be signal loss in the pass band.

The transition bandwidth is greater than zero.

The stopband attenuation is not infinite.

\section{Conclusion}

The mathematical expressions for MPS and APS are easily computed, which provide an automatic approach to evaluate the application of the comb filter in digital audiography in multirate systems. In future research, finite word length effects can be considered. The design procedure is comfortable to implement FIR decimation in Digital Signal Processing. Digital decimation filters have been widely employed in consumer electronics to simplify the analog front end, and to improve the overall digital audio system performances. In this paper efficient design techniques for FIR decimation filters have been shown. The proposed method would be useful that it can improve the decimation performance of the Comb FIR filter in DSP technology.

\section{Acknowledgements}

The authors would like to thank the Editor-in-Chief and the reviewers for their valuable comments and constructive criticisms on the manuscript which made this final contribution considerably easier for the readers to grasp.

\section{References}

[1] Crochiere, R.E. and Rabiner, L.R. (1983) Multirate Signal Processing. Prentice-Hall, Englewood Cliffs.

[2] Vaidyanathan, P.P. (1993) Multirate Systems and Filter Banks. Prentice-Hall, Englewood Cliffs. 
[3] Deng, Y., Mathews, V.J. and Farhang-Boroujeny, B. (2007) Low-Delay Nonuniform Pseudo-QMF Banks with Application to Speech Enhancement. IEEE Transactions on Signal Processing, 55, 2110-2121. http://dx.doi.org/10.1109/TSP.2007.892707

[4] Rajic, H. and Babic, H. (2004) Efficient Implementation of Sharpened CIC Decimation Filters for Software Radio Receivers. 15th IEEE International Symposium on Personal, Indoor and Mobile Radio Communications, 3, 1672-1676. http://dx.doi.org/10.1109/pimrc.2004.1368285

[5] Laddomada, M. and Mondin, M. (2004) Decimation Schemes for $\Sigma \Delta$ A/D Converters Based on Kaiser and Hamming Sharpened Filters. IEE Proceedings-Vision, Image and Signal Processing, 151, 287-296. http://dx.doi.org/10.1049/ip-vis:20040477

[6] Ghazel, A., Naviner, L. and Grati, K. (2002) On Design and Implementation of a Decimation Filter for Multistandard Wireless Transceivers. IEEE Transactions on Wireless Communications, 1, 558-562. http://dx.doi.org/10.1109/TWC.2002.805093

[7] Kim, J.T. (2005) Efficient Implementation of Polynomial Interpolation Filters or Full Digital Receivers. IEEE Transactions on Consumer Electronics, 51, 175-178. http://dx.doi.org/10.1109/TCE.2005.1405716

[8] Proakis, J.G. and Manolakis, D.G. (2007) Digital Signal Processing: Principles, Algorithms and Applications. 4th Edition, Prentice-Hall/Pearson Education, Englewood Cliffs.

[9] Wise, D.K. (2006) The Modified Chamberlin and Zölzer Filter Structures. Proceedings of 9th International Conference on Digital Audio Effects (DAFx-06), Montreal (Canada), 18-20 September 2006, 53-56.

[10] Antoniou, A. (1993) Digital Filters: Analysis, Design, and Applications. 2nd Edition, Mac-Graw Hill, New York.

[11] Li, H., Wang, Q. and Lu, C.Y. (2006) Optimal Farrow Coefficients for Timing Recovery in QAM Demodulation Receiver. 2006 IEEE Sarnoff Symposium, Princeton, 27-28 March 2006, 1-4.

[12] Altera (2009) Simplifying Simultaneous Multimode RRH Design. White Paper, WP-01097, 1-13.

[13] Milic, L. (2009) Multirate Filtering for Digital Signal Processing: MATLAB Applications. Information Science Reference, London. http://dx.doi.org/10.4018/978-1-60566-178-0

[14] Harris, F. (2009) Multirate Signal Processing for Communication Systems. Pearson Education, New Jersey.

[15] Cruz-Roldán, F., Del Campo, J.D.O., Godino-Llorente, J.I., Boquete-Vázquez, L. and Bleakley, C.J. (2010) Polyphase FIR Networks Based on Frequency Sampling for Multirate DSP Applications. Circuits, Systems, and Signal Processing, 29, 169-181. http://dx.doi.org/10.1007/s00034-009-9140-5

[16] Chaturvedi, S. and Parmar, G. (2012) Shukla, Sharpening the Response of an FIR Filter Using Fractional Fourier Transform. International Journal of Electronics and Computer Science Engineering, 1, 657-664.

[17] Mohindru, P., Khanna, R. and Bhatia, S.S. (2012) A Novel Design Technique for Variable Non-Recursive Digital Filter Based on FrFT. Elektronika Ir Elektrotechnika, 121, 89-92. http://dx.doi.org/10.5755/j01.eee.121.5.1657

[18] Mondal, K. and Mitra, S. (2012) Non-Recursive Decimation Filters with Arbitrary Integer Decimation Factors. IET Circuits, Devices \& Systems, 6, 141-151. http://dx.doi.org/10.1049/iet-cds.2011.0240

[19] Ye, H., Zhao, L. and He, Z. (2009) Study of High Performance and Efficient Decimator Filters. IET International Communication Conference on Wireless Mobile \& Computing (CCWMC 2009), Shanghai, 7-9 December 2009, 745748.

[20] Meyer-Baese, U., Rao, S., Ramírez, J. and García, A. (2005) Cost-Effective Hogenauer Cascaded Integrator Comb Decimator Filter Design for Custom ICs. Electronics Letters, 41, 158-160. http://dx.doi.org/10.1049/el:20057000

[21] Romero, D.E.T. and Dolecek, G.J. (2013) Application of Amplitude Transformation for Compensation of Comb Decimation Filters. Electronics Letters, 49, 985-987. http://dx.doi.org/10.1049/el.2013.1492

[22] Li, T. (2008) Digital Signal Processing: Fundamentals and Applications. Academic Press, New York. 


\section{Submit or recommend next manuscript to SCIRP and we will provide best service for you:}

Accepting pre-submission inquiries through Email, Facebook, LinkedIn, Twitter, etc.

A wide selection of journals (inclusive of 9 subjects, more than 200 journals)

Providing 24-hour high-quality service

User-friendly online submission system

Fair and swift peer-review system

Efficient typesetting and proofreading procedure

Display of the result of downloads and visits, as well as the number of cited articles

Maximum dissemination of your research work

Submit your manuscript at: http://papersubmission.scirp.org/ 